\title{
Rapid evolution of a retro-transposable hotspot of ovine genome underlies the alteration of $B M P 2$ expression and development of fat tails
}

\author{
Zhangyuan Pan ${ }^{1,3+}$, Shengdi Li, ${ }^{2,4}$, Qiuyue Liu', Zhen Wang ${ }^{2}$, Zhengkui Zhou' ${ }^{1}$, Ran Di $^{1}$, Xuejiao An ${ }^{1}$, \\ Benpeng Miao ${ }^{2,4}$, Xiangyu Wang' ${ }^{1}$, Wenping $\mathrm{Hu}^{1}$, Xiaofei Guo', Shenjin $\mathrm{Lv}^{3}$, Fukuan $\mathrm{Li}^{3}$, Guohui Ding ${ }^{2,4}$, \\ Mingxing $\mathrm{Chu}^{1 *+}$ and Yixue $\mathrm{Li}^{2,4^{*+}}$
}

\begin{abstract}
Background: Sheep have developed the ability to store fat in their tails, which is a unique way of reserving energy to survive a harsh environment. However, the mechanism underlying this adaptive trait remains largely unsolved.

Results: In the present study, we provide evidence for the genetic determinants of fat tails, based on whole genome sequences of 89 individual sheep. A genome-wide scan of selective sweep identified several candidate loci including a region at chromosome 13, a haplotype of which underwent rapid evolution and spread through fat-tailed populations in China and the Middle East. Sequence analysis revealed an inter-genic origin of this locus, which later became a hotspot of ruminant-specific retro-transposon named BovB. Additionally, the candidate locus was validated based on a fat- and thin-tailed cross population. The expression of an upstream gene BMP2 was differentially regulated between fat-tailed and thin-tailed individuals in tail adipose and several other tissue types.
\end{abstract}

Conclusions: Our findings suggest the fixation of fat tails in domestic sheep is caused by a selective sweep near a retro-transposable hotspot at chromosome 13, the diversity of which specifically affects the expression of BMP2. The present study has shed light onto the understanding of fat metabolism.

Keywords: Retro-transposable hotspot, BMP2, Fat tail, Sheep, Evolution

\section{Background}

Storing fat is an essential process to temporarily reserve energy, which enables animals to endure harsh environments. Sheep, a major type of livestock, have developed a unique mechanism to store fat in tails, which is present in about $25 \%$ of the global sheep population [1]. Fat-tailed sheep are commonly found in Asia and northern Africa [1]. In China, fat-tailed breeds from an ancestral lineage, known as Mongolian sheep, are widely distributed in the

\footnotetext{
* Correspondence: mxchu@263.net; yxli@sibs.ac.cn

${ }^{+}$Mingxing Chu and Yixue Li jointly supervised this work.

${ }^{1}$ Institute of Animal Science, Chinese Academy of Agricultural Sciences, Beijing, China

${ }^{2}$ Key Lab of Computational Biology, CAS-MPG Partner Institute for Computational Biology, Shanghai Institutes for Biological Sciences, Chinese Academy of Sciences, Shanghai, China

Full list of author information is available at the end of the article
}

northern area $[2,3]$. Owing to the fact that the wild ancestors of domestic sheep all have thin tails, it has been suggested that fat tails were developed following domestication as an adaptive response to preserve energy during migration and winter $[4,5]$. Fat-tailed sheep are adapted to harsh environments characterized by extreme cold [6], dryness [7], and food scarcity [8]. Excess tail fat serves as a source of energy that improves the probability of survival through the winter [8]. However, over-deposition of fat may compromise fertility and reproductive fitness $[9,10]$, thus reducing the economic value of the sheep. Consequently, the association between tail fat and reduced fertility and reproductive fitness in sheep has led researchers to investigate the genetic determinants of fat tails. Several studies have provided evidence of crucial genes influencing tail types based on single nucleotide

(c) The Author(s). 2019 Open Access This article is distributed under the terms of the Creative Commons Attribution 4.0 International License (http://creativecommons.org/licenses/by/4.0/), which permits unrestricted use, distribution, and reproduction in any medium, provided you give appropriate credit to the original author(s) and the source, provide a link to the Creative Commons license, and indicate if changes were made. The Creative Commons Public Domain Dedication waiver (http://creativecommons.org/publicdomain/zero/1.0/) applies to the data made available in this article, unless otherwise stated. 
polymorphism (SNP) marker panels $[4,5,11]$. However, a lack of agreement regarding the affected loci between different fat-tailed populations suggests a complicated mechanism underlying this adaptive trait. Moreover, the application of marker panels is efficient in searching for regions with signals of selection, but often has a restricted power to detect the causative genes or variants.

In the present study, we provide evidence of a selective sweep in Chinese fat-tailed sheep based on whole-genome sequencing of 49 Mongolian (fat-tailed), 30 Tibetan (thin-tailed) and 10 European (thin-tailed) sheep. We evaluated the signatures of positive selection, including a top signal at chromosome 13 identified by independent studies but still with conflicting understandings of causative genes $[4,11]$. We studied the sequence origin of this locus by inter-species comparisons and analyzed gene expression patterns in different tail types. The aim of this study was to understand the lineage evolution of domestic sheep and clarify the potential mechanism underlying their essential phenotypes in adaptation.

\section{Results}

Analysis of a selective sweep based on whole-genome sequencing of domestic sheep

To elevate our understanding of ovine lineage evolution, we analyzed the abundant genetic variants ( 40 million) generated from whole-genome sequencing (WGS) data of 89 sheep (Materials and Methods). The genetic relationship between these individuals has been well described elsewhere [12], suggesting that 49 out of the 89 samples from five Chinese Mongolian sheep breeds (MGS) form a major ovine lineage in China (the genetic distances between these breeds are small), distinct from the Chinese Tibetan sheep breeds (TBS) and European sheep breeds (EUS). The phylogenetic structure of these lineages (Fig. 1a) is consistent with the mito-genomic

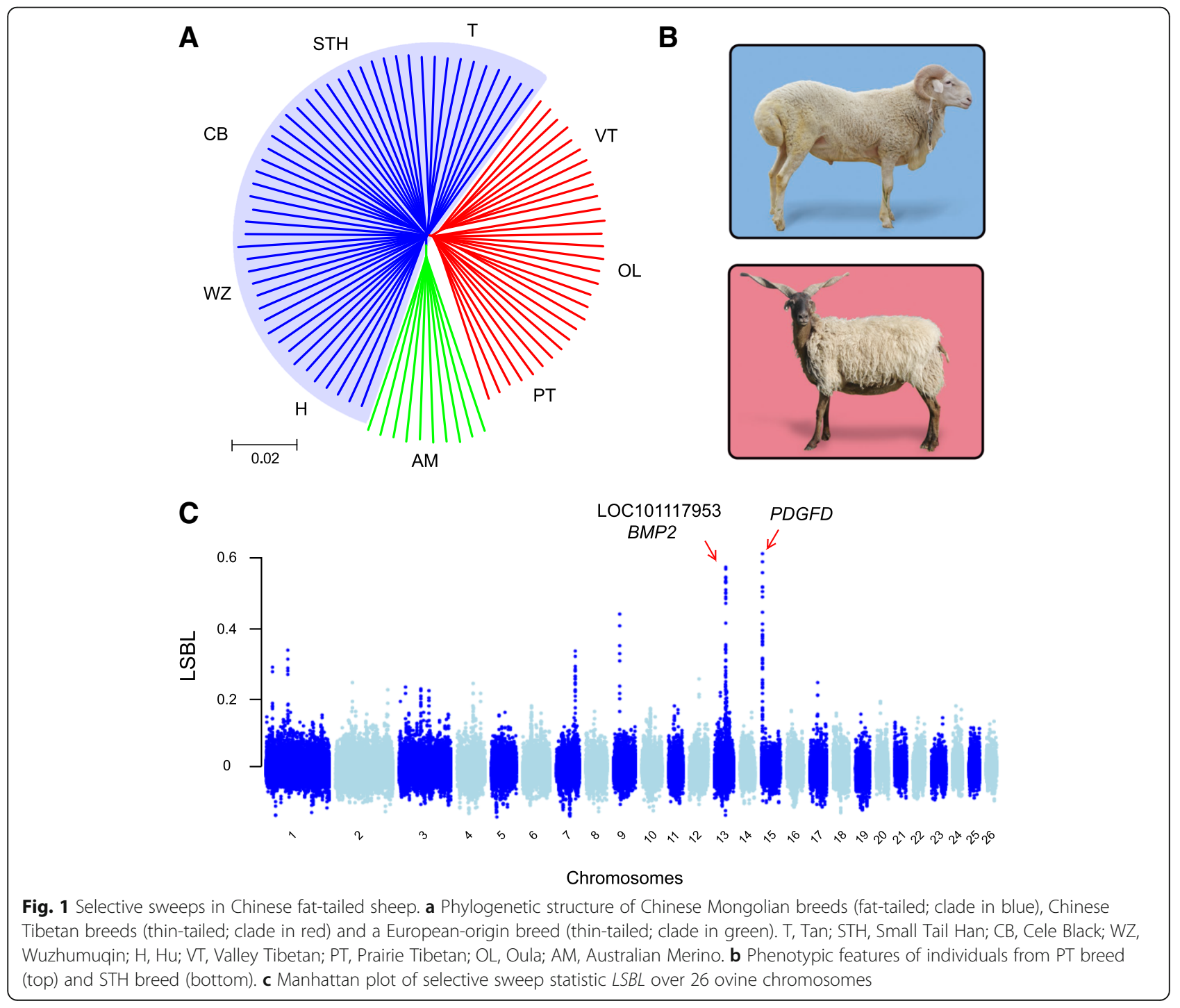


evidence of ovine migrating trajectory from Eurasia to China [13].

We next calculated the lineage-specific branch length $(L S B L)$ [14, 15] of MGS on a sliding-window basis (Materials and Methods). The statistic measures the extent of population differentiation in the MGS clade by comparing them with TBS and EUS, and is therefore a powerful indicator of a recent selective sweep. As displayed in the Manhattan plot (Fig. 1c), two highly significant signals of high $L S B L$ values $\left(L S B L_{\text {top }}=0.574\right.$ and $\left.L S B L_{\text {top }}=0.613\right)$ were found in chromosome 13 and 15 . Both signals were supported by extensive numbers of high- $L S B L$ windows (Additional file 1: Table S1) because of a hitchhiking effect on the variants in linkage with the selected sites. In particular, a sweep signal at chromosome 13 was consistent with the results from independent studies of fat-tailed populations $[4,11]$, nevertheless its causative event is controversial because the region contains multiple genes. Specifically, Moioli et al. [4] suggested the bone morphogenetic protein 2 (BMP2), while Wei et al. [11] suggested the protein phosphatase 1 catalytic subunit gamma $(P P P 1 C C)$ to be causative (it is a paralogue of the original PPP1CC gene at ovine chromosome 13 with NCBI gene identifier LOC101117953).

\section{Genomic region encompassing LOC101117953 underwent a selective sweep in Mongolian sheep}

Due to the genetic hitchhiking among variants in close proximity, it is often difficult to assess the true causative mutations underlying the alterations of phenotype and fitness. As WGS of ovine population provided a novel opportunity to study their genomic architectures at higher resolution, we further analyzed the inter- and intra-population diversities of the highly significant sweep regions (Fig. 2 and Additional file 2: Figure S1). An obvious peak point of $L S B L$ values was observed at position $\sim 49 \mathrm{M}$ bases $(\mathrm{Mb})$ on chromosome 13 (Fig. 2a). As expected, the signal strength was attenuated by increasing distance to the peak-point, and became insignificant after $\sim 0.5 \mathrm{Mb}$ extension on either side. Two protein-coding genes were annotated in this region: a newly characterized gene LOC101117953 is in the center of high- $L S B L$ block, while $B M P 2$ is upstream to it near the border (Fig. 2b). A region encompassing LOC101117953 of $\sim 250 \mathrm{~kb}$ exhibited excessive population differentiation (measured by genetic distance $d_{\mathrm{xy}}$ ) between MGS vs. EUS and MGS vs. TBS, but not TBS vs. EUS (Fig. 2b). Moreover, the region also showed a depletion of intra-population diversities (measured by heterozygosity $Z H_{\mathrm{P}}$ ) in MGS (Fig. 2b). These findings collectively indicate that the Mongolian lineage has experienced a recent sweep over the locus encompassing LOC101117953.
We then questioned whether this locus could explain fat-tailed phenotypes in ovine populations outside of China. By comparing the variant distributions among sheep from China, Iran (IROA) and northern Africa (MOOA), we noticed a haplotype sweeping through the MGS population was also present in IROA at high frequency (Fig. 2c), which is in agreement with the fact that many Iranian breeds have fat tails [1]. Although it is unknown whether the locus is also under selection in other fat-tailed populations, the data here suggested the alternative haplotype has been derived in domestic sheep before their spread in China.

\section{LOC101117953 is derived from retro-transposable event}

As a newly characterized gene, LOC101117953 is annotated as a paralogue of PPP1CC based on sequence similarity. Although dosage effect (assuming LOC101117953 has a function similar to that of PPP1CC) is a possible mechanism underlying how LOC101117953 may yield a fitness advantage to the fat-tailed ovine lineage, it is not completely convincing due to a lack of knowledge about this novel gene. To consider more possibilities, we performed analysis to categorize and date the origin of LOC101117953, which is essential for understanding its functionality. Intriguingly, the alignment of two paralogue gene sequences (with introns) suggested a retro- $t$ ransposable origin of LOC101117953 from PPP1CC, evidenced by clear intron losses in the derived locus (Fig. 3a). Moreover, as loss of introns can be attributed to either retro-transposition or artifacts from RNA contamination in the sequencing library, primers were designed to capture and validate the DNA sequence of LOC101117953 based on the classical Sanger sequencing method $[16,17]$. This experiment confirmed the existence of gene duplication (Additional file 2: Figure S2A). In addition, there is an artificial intron of LOC101117953 (homologous to the 4th intron of PPP1CC) in the reference genome compared with ensuing validation (Additional file 2: Figure S2B), which is a likely assembly error due to the limitation of short-read sequencing.

To date the origin of LOC101117953, which has now been proven as the retro-copy of PPP1CC (r-PPP1CC), nucleotide BLAST [18] was performed against six vertebrate genomes making the ovine $\mathrm{r}-P P P 1 C C$ sequence as the query. The orthologues were determined by both sequence similarity and shared synteny among species: true $\mathrm{r}-P P P 1 C C$ orthologues were located at the intergenic region between $B M P 2$ and HAO1 (IBH region). Among all the searched vertebrates, orthologs of r-PPP $1 C C$ were merely found in two Caprinae species: sheep and goat (sub-family Caprinae is inside ruminant family Bovidae). By sequence alignment of this region between sheep and cattle, the insertion point of the retro-transposon was clearly observed (Fig. 3b), suggesting 


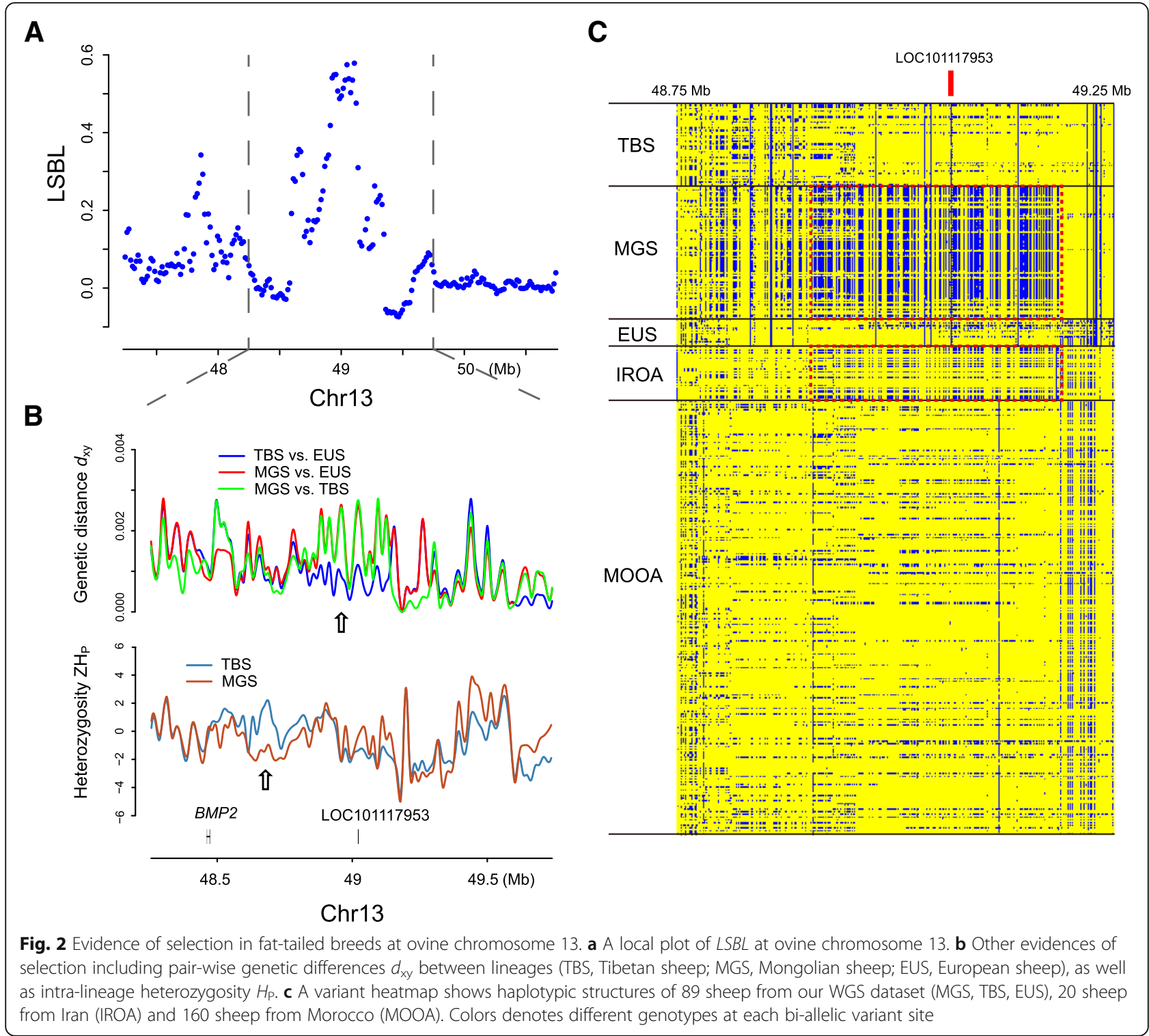

the exact time of the retro-transposable event after the coalescence of family Bovidae ( 26.5 Ma, estimated by TimeTree [19]) and before that of sub-family Caprinae $(\sim 12.5 \mathrm{Ma})$. Moreover, the phylogenetic structure of all $P P P 1 C C$ homologues confirmed the same emergence time of $\mathrm{r}-P P P 1 C C$ within the sub-clade comprising sheep and goat (Fig. 3c). Taken together, these findings demonstrate that LOC101117953 ( $\mathrm{r}-\mathrm{PPP} 1 C \mathrm{C})$ is a novel gene copy derived from a retro-transposable event, which has been present in part of the ruminant family.

\section{$\mathrm{IBH}$ region of sheep is a ruminant-specific retro-transposable hotspot}

Although a single retro-transposable event can occur in isolation, retro-transposons derived from processed RNA are often clustered at common loci of the genome [20].
Thus, we questioned whether r-PPP1CC was the only retro-copy of actively transcribing genes in the IBH region. To test this, the genomic sequence from $48.5 \mathrm{Mb}$ to $49.5 \mathrm{Mb}$ of chromosome 13 was compared with the ovine RNA database to search for potential RNA-derived sequences, and afterwards these sequences were aligned with their normal paralogues to confirm the absence of introns (Fig. 4a). In total, eight candidate RNA-derived sequences were identified, in which six were confirmed as retro-copies with clear evidence of intron deletion. (Additional file 2: Figure S3). Among the six retro-copies, five were from different chromosomes ( $\mathrm{r}-P P P 1 C C, \mathrm{r}-B L O C 1 S 5$, r-LOC105603412, r-LOC105615525, r-LOC105611745), and one was from a distant location of chromosome 13 (r-RBM17) (Fig. 4b). Given these, the IBH region of sheep seems to be a hotspot of retro-transposable events, which 


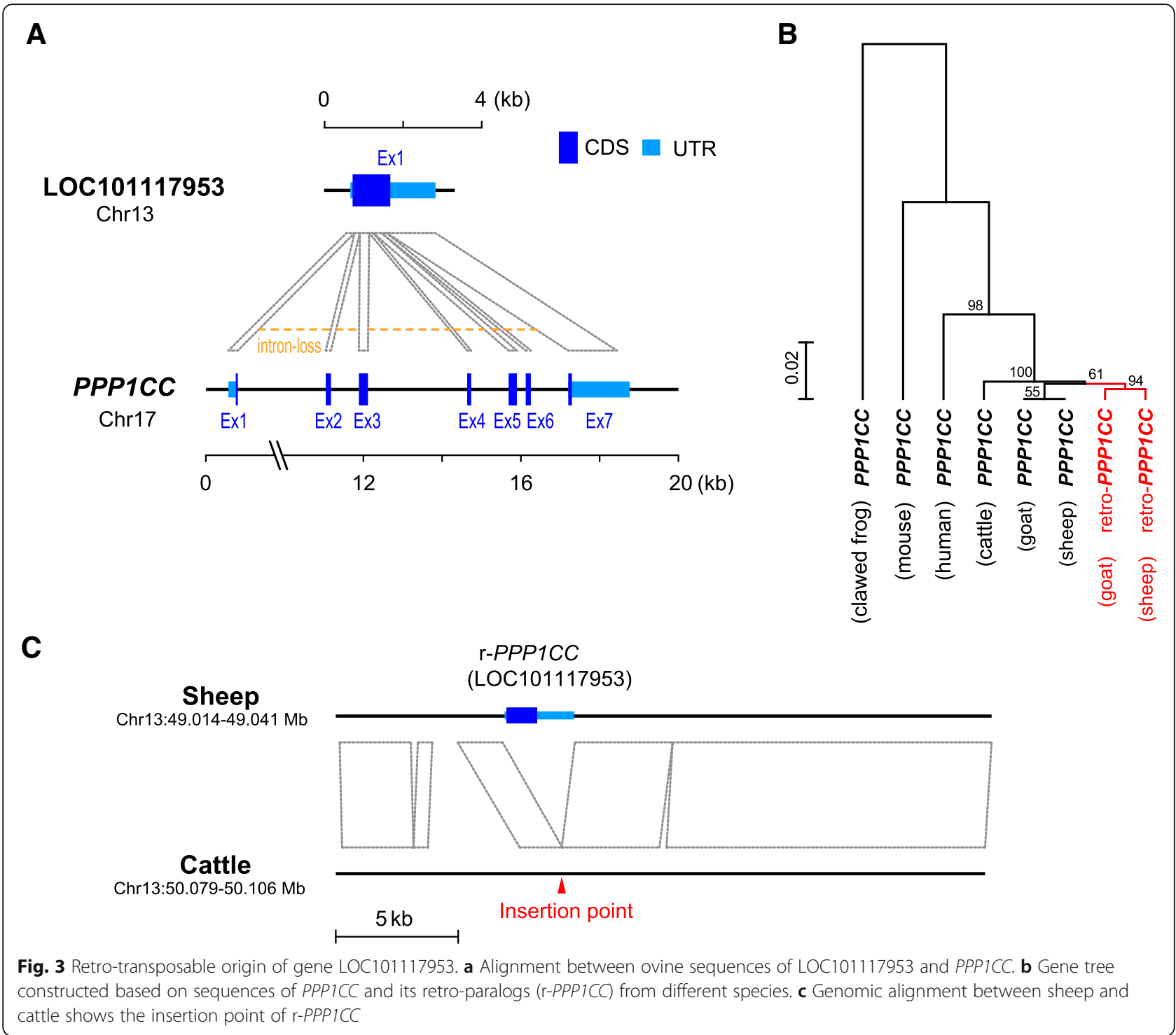

comprises six or even more undetected gene copies derived from intron-less RNA.

There are various types of retro-transposable elements (RTE) in mammals, including long terminal repeat (LTR) and non-LTR retro-transposons [21]. We now have proven that at least six retro-copies of processed transcripts were clustered at the IBH region of ovine genome, and it is therefore intriguing to know which type of RTEs have contributed to anchoring these retro-transposons. By annotating the $1 \mathrm{Mb} \mathrm{IBH}$ region with known interspersed repeats of mammal genomes (Table 1), an impressively high proportion of the sequence $(21.32 \%)$ was identified as a ruminant-specific long interspersed nuclear element (LINE) named BovB [22]. Density of this repeat sequence ranged from about 10 to $45 \%$ when scanning the whole region with a 50-kb sliding window (Fig. 4c). In addition to this, L1 repeats known as the dominant retro-transposon type in mammals [23] comprised $14 \%$ of the locus, while other types including short (S) INEs and LTR elements comprised 7.95 and 5.07\% (Table 1).

To test whether IBH region is a favorable location of RTE insertion, we compared the density of each retro-transposon type between IBH vs. random loci $(n=10)$. Impressively, LINEs comprised an obviously higher proportion in IBH region than in random locations (IBH: 36.29\%; random: $22.76 \pm 4.09 \%$ ), due to a profound enrichment of BovB repeats (IBH: $21.32 \%$; random: $10.07 \pm 3.45 \%$ ). By contrast, other transposon types (SINEs, LTRs, DNA elements) showed either less or equal occupations of IBH compared to random locations (Fig. 4d).

Given these, BovB seems to be the most likely RTE type which has mediated frequent retro-transposon insertions into IBH region comprising over six retro-copies of gene 


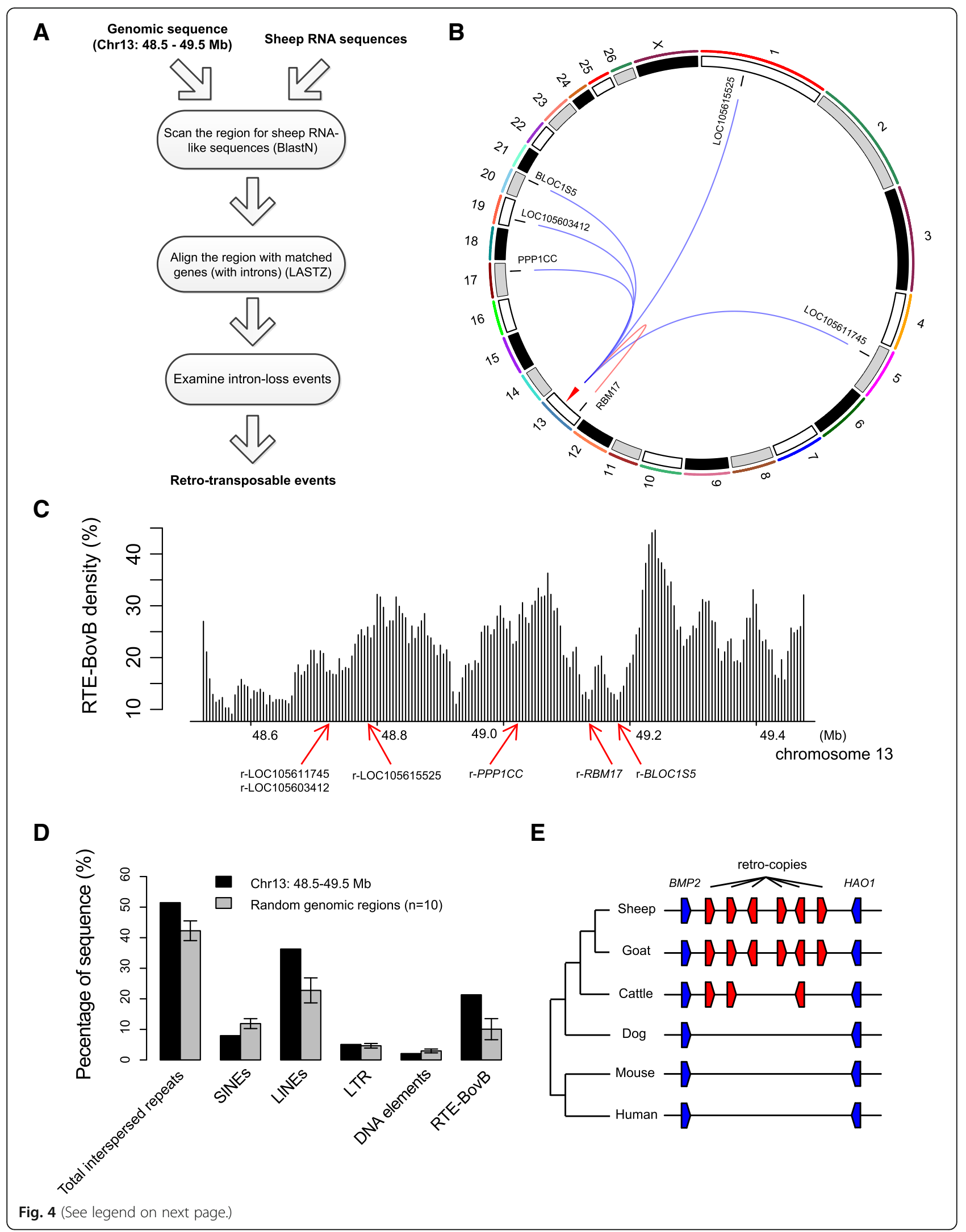


(See figure on previous page.)

Fig. 4 Identification of retro-transposable events at IBH region. a Scheme for identifying retro-transposable events at IBH region, specifically from 48.5 to $49.5 \mathrm{Mb}$ at chromosome 13. b A circular plot of ovine chromosomes and six retro-transposable events detected at IBH region. c Slidingwindow-based densities of the RTE BovB, calculated from the prediction of functional repeats by RepeatMasker. $\mathbf{d}$ Comparison of RTE occupation between IBH and random genomic regions $(n=10)$. Error bars denotes standard deviations. $\mathbf{e}$ The positions and emergence time of six retro-copies estimated based on a cross-species comparison

transcripts. Moreover, this specificity is further evidenced by a consistent chronological order between the initial emergence of RTE and the subsequent retro-transpositions. BovB was proposed to have been horizontally transferred from squamata to ruminants $[22$, 24], therefore BovB-related retro-transposable events should not precede the coalescent time of ruminant family and the retro-copies should be present merely in ruminant species. This is exactly the case for the retro-transposons identified in IBH, where sheep and goat carry all six retro-copies, cattle carry three of them, and none is present in other vertebrate species (Fig. 4e).

\section{Haplotype of the IBH region predicts tail phenotypes in a hybrid population}

Though we have corroborated that tail types of Chinese sheep are highly consistent with their IBH genotypes, it is possible that the selective sweep at the IBH region may affect other phenotypes distinguished between fat-tailed and thin-tailed populations. This is often the case when the studied phenotype is correlated with population structures, which means the genetic differentiation between groups may have various explanations. To fully address this question, we tested whether IBH haplotype could predict tail phenotypes within a hybrid population with diverse shapes of tail (Additional file 2: Figure S4). Due to chromosomal recombination, only the association between the causative locus and tail phenotypes will be steadily passed on over generations, thus enabling the exclusion of loci affecting other phenotypic outcomes.
The hybrid population we used comprises 116 individuals (28 females and 88 males), in which four genetic markers were genotyped (two for IBH region, one for $B M P 2$ and one for PDGFD, see Additional file 1: Table S2 and S3). Tail length and width, which are known to correlate with fat storage capability, were measured for all individuals. Linear regressions were performed to evaluate the association between genetic markers and tail phenotypes, based on adjustment of age and gender (Additional file 1: Table S4). Intriguingly, two IBH markers (which are in complete LD) showed dramatically strong association with tail length and width (Fig. 5). The trend best fits an additive genetic model, where each mutated allele accumulatively confers $\sim 3.3$ $\mathrm{cm}$ length decrease $\left(P\right.$-value $\left.=1.57 \times 10^{-15}\right)$ and $\sim 3.5$ $\mathrm{cm}$ width increase $\left(\mathrm{P}\right.$-value $\left.=1.51 \times 10^{-27}\right)$. With this "smoking gun", we demonstrated that the IBH genotype in Mongolian sheep confers their short and fat tails.

The genotype of a $B M P 2$ missense variant was slightly correlated with tail width without considering covariates (additive model: P-value $=0.058$; dominant model: P-value $=0.025$ ), nevertheless the association became insignificant after adjustment for age and gender (additive model: $\mathrm{P}$-value $=0.167$; dominant model: $\mathrm{P}$-value $=0.245)$. PDGFD marker shows a moderate correlation with tail width after adjustment (additive model: P-value $=0.0108$ ), whilst it seems not to affect tail length (additive model: P-value $=$ 0.149). This result suggests $P D G F D$ does not has a strong effect on tail phenotypes: although it shows the highest $L S B L$ value in fat-tailed sheep (Fig. 1c), its correlation with tail types is poorly detected in hybrid populations because

Table 1 Transposable elements detected in $1 \mathrm{Mb}$ IBH region

\begin{tabular}{|c|c|c|c|c|}
\hline Element class & Subclass & Most frequent elements & Number of elements & Percentage on region \\
\hline \multirow[t]{2}{*}{ SINES } & MIRs & MIR, MAR1 & 134 & $1.26 \%$ \\
\hline & tRNA \& core-RTE & Bov-A2, Bov-tA & 518 & $6.69 \%$ \\
\hline \multirow[t]{4}{*}{ LINES } & L1 & L1 & 248 & $14 \%$ \\
\hline & L2 & L2 & 48 & $1.18 \%$ \\
\hline & CR1(L3) & L3 & 7 & $0.11 \%$ \\
\hline & RTE-BovB & BovB & 304 & $21.32 \%$ \\
\hline \multirow[t]{4}{*}{ LTR elements } & ERVL & ERVL, LTR16 & 32 & $1.99 \%$ \\
\hline & ERVL-MaLRs & MLT1 & 36 & $0.91 \%$ \\
\hline & ERV classl & ERV1, ERV54, LTR1 & 29 & $1.19 \%$ \\
\hline & ERV classll & BTLTR1 & 75 & $0.89 \%$ \\
\hline DNA elements & & MERs, hAT-Charlie, TcMars-Tigger & 98 & $2.09 \%$ \\
\hline
\end{tabular}




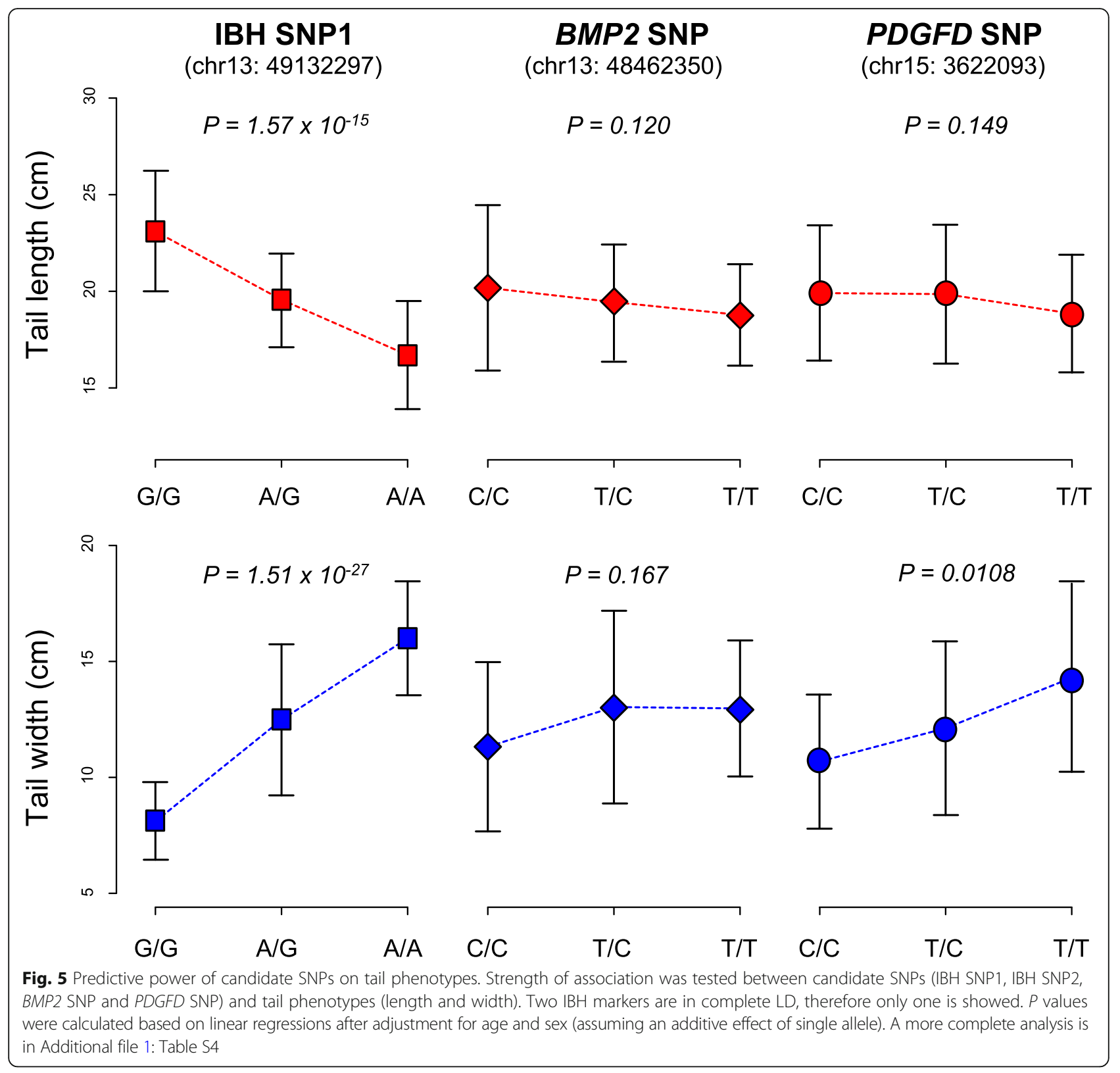

random chromosomal recombination "diluted" the linkage between distant loci.

Inference of the causative gene by gene expression profiles Given that LOC101117953 is a retro-copy of PPP1CC, we assume it is less likely to be the causative gene for tail phenotypes, although its gene body is located with the $L S B L$ peak. The mechanism of retro-transposition means that most retro-copies are pseudo-genes, because the promoter regions are often not transcribed and therefore not transposed with RTEs [20]. To test whether LOC101117953 is a pseudo-gene, we performed quantitative PCR (qPCR) in 17 tissues of thin-tailed and fat-tailed sheep (Materials and Methods). Expression of LOC101117953 was not detected (Additional file 2: Figure S5). Moreover, the putative protein sequence of LOC101117953 was truncated into 188 amino acids (AA) by a novel stop codon compared with the 323 AA protein of PPP1CC (Additional file 2: Figure S6). A neutral test based on non-synonymous vs. synonymous mutation rate ratio $(\omega)$ for the PPP1CC gene tree (Fig. 4b) revealed a much stronger trend of purification selection in the clade of PPP1CC $(\omega=0.00562)$ than $\mathrm{r}-P P P 1 C C$ $(\omega=0.1811)$. Additionally, no statistical significance $(P=0.154)$ was detected to reject neutral evolution of r-PPP1CC clade (Materials and Methods, Additional file 1: Table S6). These results suggested that LOC101117953 is 
not likely to possess essential functions, either at transcript or protein level.

We next questioned whether two protein-coding genes $B M P 2$ and $H A O 1$, based on which the borders of IBH were defined, were relevant to tail phenotypes. Although the variant under selection is unlikely to be a protein-altering or intronic mutation within their gene bodies (because they are too distant to the sweep region), we tested the hypothesis whether $B M P 2$ and $H A O 1$ gene expression was altered because of the sweep. Surprisingly, BMP2 but not HAO1, showed different tissue expression profiles between fattailed and thin-tailed groups (Fig. 6). By quantitative PCR, $B M P 2$ expression differences were detected in 5 out of the 17 tissue types, in a condition-dependent manner: The fat tailed group is correlated with enhanced $B M P 2$ transcript levels in perirenal adipose, tail adipose and adrenal gland, but with suppressed expressions in corpus and cornua uterus (Fig. 6a). We repeated the experiments in adipose and uterine tissues with additional biological replicates ( $n=8$ for each tail type), the result of which corroborated our finding and was supported by statistical significance (Fig. 6b). BMP2 is known to induce both osteoblastic and adipocytic differentiation of pluripotent stem cells [25-28], thus it seems rational that fat-tailed sheep exhibit a $\sim 3$-fold higher $B M P 2$ expression in their tail adipose tissues, consistent with a better capability of fat storage. On a different note, $B M P 2$ also plays an important role in uterine decidualization [29-31], therefore the expression pattern of BMP2 suggests that fail-tailed phenotype might be accompanied with alterations in uterine functions, which remains to be investigated.

\section{Discussion}

Various animal models have been developed to understand fat metabolism and the related disorders in humans. Abnormal body fat distribution is a common risk factor of various prevalent diseases [32-36]. Sheep, as a world-widely distributed livestock, have developed phenotypic variations in fat storage ability during their domestication, and therefore possess great potential to become a new model organism for studying fat deposition mechanically. Understanding the natural machinery in the sheep tail, which has evolved to accommodate massive deposits of lipid, could be the key to resolve how fat distribution is regulated across tissues.

Positive selection is a critical driving force for organisms to evolve new functions. A handful of methods have been developed to detect positive selection by measuring a selective sweep of genetic diversities [37]. In most applications, the aim is to determine the causative variants (or genes) that affect fitness. However, this is challenged by numerous confounding factors (e.g. genetic hitchhiking, population admixture) in practice. Here we showed that in sheep a selective sweep at the IBH region of chromosome 13, which has been suggested as the determinant of fat deposition and tail type differences [4, 11], is more complex than previously expected. Based on WGS of domestic sheep, we revisited the regions under selective sweep in Mongolian ovine populations. We provided novel evidence to show that the IBH region encompassing LOC101117953 has experienced a recent sweep in fat-tailed sheep. Moreover, data from a hybrid ovine population, for the first time, corroborated the hypothesis that the IBH genotypes confer tail differences in sheep.

Retro-transposable elements comprise a large fraction of the eukaryotic genomes. These genomic elements are considered to be the "seeds of evolution", because they have played an important role in molecular evolution [38]. Here our data analyses show that the IBH region serves as a hotspot of BovB-related retro-transpositions, which has gradually accumulated after the first introgression of BovB elements into the genome of ruminant ancestor (Fig. 7). These results also corroborate the fact that ruminant-specific RTEs are essential to the generation of novel gene copies on sheep genome. Moreover, a higher insertion frequency of species-specific RTEs compared with random loci suggests a role in species-specific functions [39]. Although the structural alterations introduced by active retro-transposons are usually more deleterious than single nucleotide substitutions, their frequent occurrences in hotspots provide more variations to be favored by natural selection. Since the mutation rate is relatively constant within a species, enrichment of retro-transposable variations potentially enables the species to rapidly evolve novel functions within a short time.

Another key question our study has addressed is the mechanism by which the selective sweep near LOC101117953 has affected fat deposition in sheep tails. Most retro-copies are considered non-functional (pseudo-genes), which significantly contribute to the genomic evolution but are not important for survival [39], so it is surprising that the IBH locus has received intense positive selection during the evolution of fat-tailed sheep. Although no evidence of transcription or potential protein function of LOC101117953 was found, our finding suggests the distant $B M P 2$ gene is differentially expressed between fat-tailed and thin-tailed sheep in many tissues, including tail adipose. Thus, we hypothesize that the high expression of $B M P 2$ in tails is likely to be the cause of tail fat deposition in sheep, which is well supported by the function of $B M P 2$ to induce commitment of stem cells into adipocytes [25-28]. A recent report also showed that fat tails are correlated with increasing number and size of adipocytes [40], where BMP2 could play a role. Intriguingly, human $\mathrm{HiC}$ data (ESC cell) suggests that BMP2 interacts frequently with its 


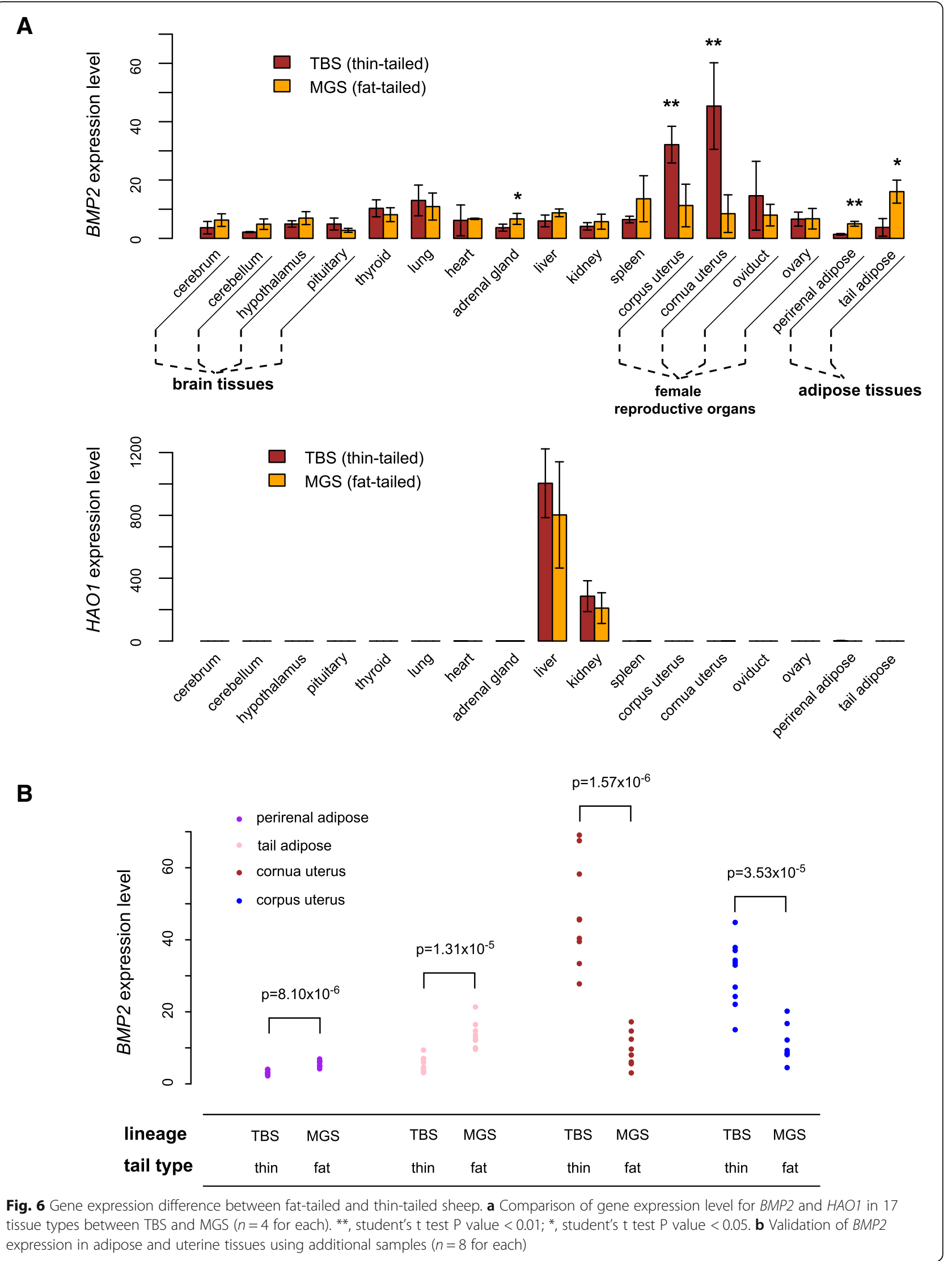




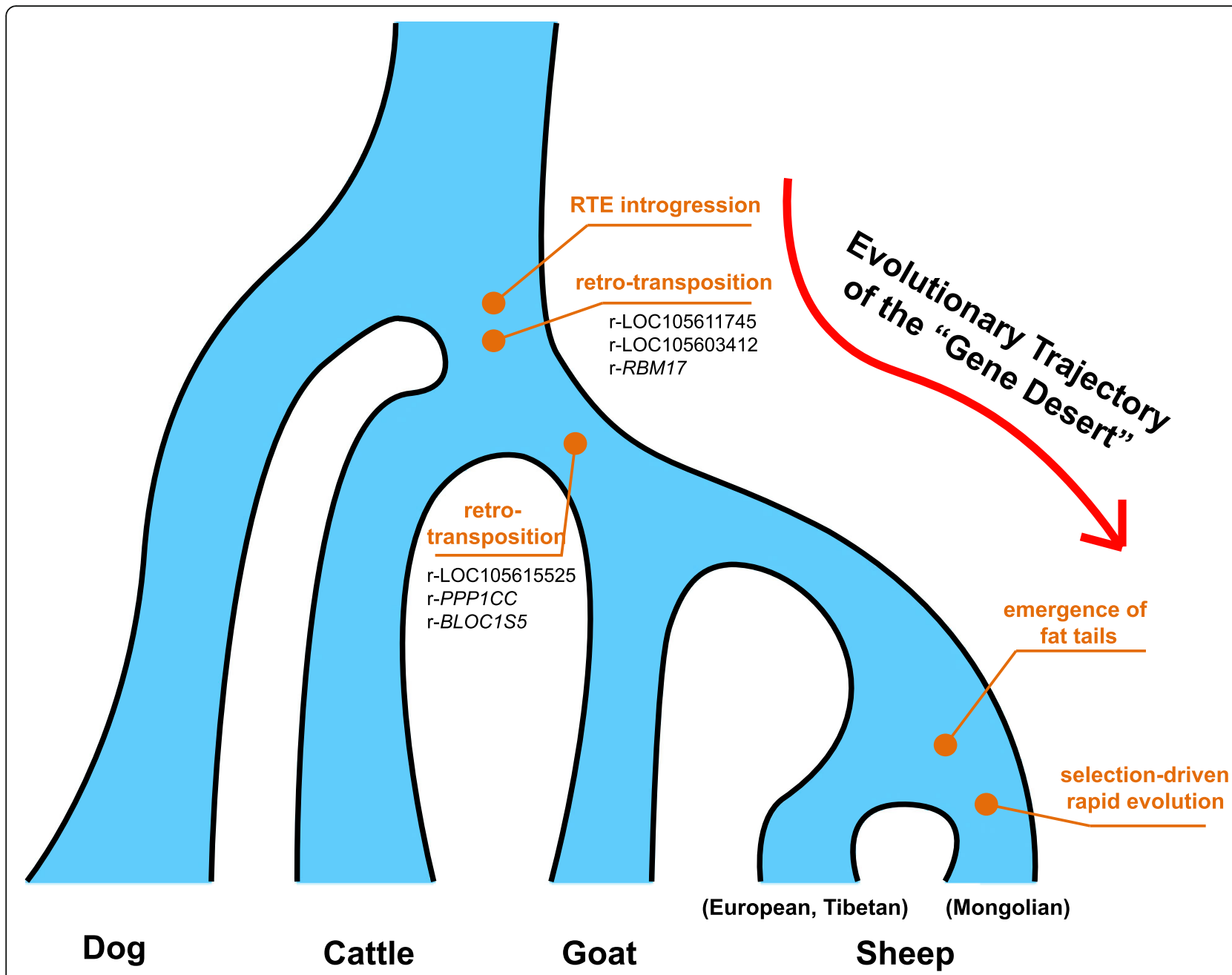

Fig. 7 Evolutionary trajectory of $\mathrm{IBH}$ region as a target of recent selection in fat-tailed sheep. The river of life summarizes from upstream to downstream the timings of several key events, which contributed to the evolution of IBH sequence, as well as its recent sweep in fat-tailed sheep

downstream regions compatible with a similar location as LOC101117953 in sheep. However, it's possible that chromatin architecture changes in sheep as consequence of the BovB retron accumulation, therefore more studies using sheep data are necessary for clarifying the role of LOC101117953 in the differential expression of $B M P 2$.

Surprisingly, $B M P 2$ is significantly down-regulated in the uterus of fat-tailed females. This unexpected finding raises the question of whether the advantageous variants for fat reservation in sheep are directly associated with their reproductive traits. On the surface, this is seemingly the case, because many fat-tailed lineages $(\mathrm{CB}, \mathrm{H}$, STH) are well known for their fertility [41]. Barbarine sheep, a breed in Tunisia, is well adapted to an environment characterized by seasonally fluctuating availability of feed resources, mainly because of its high fertility and fat tail. $B M P 2$ was also involved in mouse decidualization, a process required for implantation of the embryo and is driven by estrogen and progesterone receptors [42]. Furthermore, one report showed that BMP2 enhanced oestradiol production in granulosa cells cultured in vitro [43]. Hence, $B M P 2$ might play a role in the estrous cycle of sheep to affect fertility by regulating oestradiol production, though the BMP2 expression levels in the reproductive tract have not yet been determined. However, to fully address this question, future work is required including a comprehensive measurement of reproductive traits in various populations.

\section{Conclusion}

In summary, our data suggest that the ovine genome has encountered a recent selective sweep at the IBH locus, which confer the ability of reserving fat in tails. Comparative analysis reveals the IBH locus as a ruminant-specific retro-transposable hotspot, which plays an important role in ovine lineage evolution. Gene expression quantification indicates that the retro-gene near the sweep is poorly 
functional, but the locus potentially serves as a regulatory element that shifted the expression of distant BMP2 gene. The present study has filled a gap in the understanding of adaptive evolution of sheep tails and highlights the importance of species-specific RTE in advancing the emergence of species-specific adaptive traits. In addition, our results provide new insight into fat metabolism and novel opportunities for developing therapies for complex metabolic diseases.

\section{Methods}

\section{Variant calling from whole-genome sequencing data}

Genomic variants of 89 sheep were extracted from our previous collection of sheep whole-genome sequences $[12,44]$, of which 10 individuals from Bayinbuluke breed were not used because of population admixture. Raw read processing and mapping were performed as previously described [12]. An updated variant calling protocol was applied to detect single nucleotide substitutions and short insertions/deletions [45]: 1) use GATK [46] for base quality recalibrations, insertion/deletion realignment and variant discovery; 2) genotyping was performed across all 89 samples simultaneously. Finally, BEAGLE v4.1 [47] was used to impute missing genotypes using parameter "-gtgl".

Variant files for Iranian $(n=20)$ and Moroccan sheep $(n$ $=160$ ) in vcf format were downloaded from the NextGen project webpage (http://projects.ensembl.org/nextgen/).

\section{Phylogenetic tree construction}

The variant data was pruned by PLINK v1.07 [48] with parameter "--indep-pairwise 5050.2 " in order to generate a subset of SNPs. Pruned variants were then used to calculate the identity-by-state (IBS) distance matrix of 89 individuals using the "--genome" option. Phylogenetic tree was constructed by PHYLIP [49], using the Neighbor Joining (NJ) algorithm.

\section{Selective sweep analysis}

The lineage-specific branch length $(L S B L)$ statistic was used to search for regions that underwent a selective sweep in MGS. Calculation of $L S B L$ in MGS $\left(L S B L_{M}\right)$ based on a 30-kb sliding window with a 15-kb step was performed by our in-house perl script, which can be broken down into two steps: (1) calculate the fixation index $F I$ (or $F_{\mathrm{ST}}$ ) between MGS vs. TBS, MGS vs. EUS and TBS vs. EUS, which are represented by $F I_{M,}, F I_{M, E}$ and $F I_{T, E}$; (2) calculate $L S B L_{M}$ using formula:

$$
L S B L_{M}=\frac{F I_{M, T}+F I_{M, E}-F I_{T, E}}{2}
$$

Next, we used two window-based statistics: (1) pairwise nucleotide differences $d_{x y}$; and (2) heterozygosity $Z H_{\mathrm{P}}$ to visualize the selective sweeps at chromosome 13 and 15. Given two populations $x$ and $y$, and a genomic region with $n$ variants, we first calculated in each population the major and minor allele frequencies of the $k$ th variant $f_{k, \text { major }}$ and $f_{k, \text { minor }}$. Then, $d_{x y}$ was calculated as:

$$
\begin{aligned}
d_{x y} & =\sum_{k=1}^{n} f_{k, x, \text { major }} \sum_{k=1}^{n} f_{k, y, \text { minor }} \\
& +\sum_{k=1}^{n} f_{k, y, \text { major }} \sum_{k=1}^{n} f_{k, x, \text { minor }}
\end{aligned}
$$

And the intra-population heterozygosity $H_{\mathrm{P}}$ was calculated as:

$$
H_{\mathrm{P}}=\frac{2 \cdot \sum_{k=1}^{n} f_{k, \text { major }} \sum_{k=1}^{n} f_{k, \text { minor }}}{\left(\sum_{k=1}^{n} f_{k, \text { major }}+\sum_{k=1}^{n} f_{k, \text { minor }}\right)^{2}}
$$

Subsequently, $H_{\mathrm{P}}$ was transformed into the standardized $Z H_{\mathrm{P}}$ :

$$
Z H_{\mathrm{P}}=\frac{H_{\mathrm{P}}-\mu}{\sigma}
$$

In the formula, $\mu$ denotes the mean and $\sigma$ denotes the standard deviation of $H_{\mathrm{P}}$.

Experimental validation of LOC101117953 gene sequence Two pairs of primers (Additional file 1: Table S5) were designed for cloning the complete DNA sequence of LOC101117953. The primers targeted two overlapped fragments of $1487 \mathrm{bp}$ and $2978 \mathrm{bp}$ as displayed on the electrophoresis gel (Additional file 2: Figure S2A), which were subsequently assembled into a 3458-bp complete sequence and were compared with the reference genome (Additional file 2: Figure S2B).

\section{Identification of retro-transposable events and retro-transposable element analysis}

The process of identifying retro-transposable events was described in Fig. 4a. First, BLASTN search, setting the 1 $\mathrm{Mb} \mathrm{IBH}$ sequence as query, was performed against the reference RNA sequences of sheep (https://blast.ncbi.nlm. nih.gov/Blast.cgi? $\quad$ PROGRAM = blastn\&PAGE_TYPE = BlastSearch\&LINK_LOC = blasthome; database set as "Reference RNA sequences" and organism set as "sheep"). RNA sequences with $>500$ bp aligned to IBH and $>80 \%$ identity were chosen as candidate duplication events. Next, their complete gene sequences (with introns) were extracted and re-aligned with IBH sequence using LASTZ v1.04.00 [50]. Finally, we used dot plots to visualize their alignments with IBH region to confirm the loss of intron sequences (Additional file 2: Figure S3). 
RepeatMasker (http://www.repeatmasker.org) was used to annotate RTEs on the IBH sequence, with DNA source chosen as "mammal".

\section{Genotype-phenotype association study}

Venous jugular blood samples were collected from 116 sheep from a crossbreed (about 4-6 generations) between STH sheep (fat-tailed) and Dorper sheep (thin-tailed) in Lan Ling Shun Yuan Farm of Shandong province. Whole blood was used for DNA extraction by phenol-chloroform method. SNP markers which represent MGS haplotypes were chosen based on their single-nucleotide $L S B L$ value: a high value suggests MGS carries distinct genotypes at the site compared with TBS and EUS. Four SNPs representing three genomic regions (IBH, BMP2 and PDGFD) were genotyped in all 116 samples (Additional file 1: Table S2 and S3).

Polymerase chain reactions (PCR) were performed in volumes of $20 \mu \mathrm{L}$ including $10 \mu \mathrm{L}$ Taq PCR MasterMix (TIANGEN, Beijing), $0.5 \mu \mathrm{L}$ each of forward and reverse primer, $1 \mu \mathrm{L}$ DNA, and additional $\mathrm{ddH}_{2} \mathrm{O}$. The program was run on a Mastercycler 5333 (Eppendorf AG, Hamburg, Germany) according to the following procedure: 5 min at $95^{\circ} \mathrm{C}$ for initial denaturation, 32 cycles of $30 \mathrm{~s}$ each at $95^{\circ} \mathrm{C}, 30 \mathrm{~s}$ at the appropriate temperature as shown in Additional file 1: Table S5, $30 \mathrm{~s}$ at $72{ }^{\circ} \mathrm{C}$, and final extension for $8 \mathrm{~min}$ at $72^{\circ} \mathrm{C}$. The PCR products were validated by electrophoresis on $1.5 \%$ agarose gels (Promega, Madison, WI, USA) and sequenced by Sangon Biotech (Shanghai) Co., Ltd.

Association test between tail phenotypes (tail length and width) and individual genotypes were performed using linear regressions (with our in-house $\mathrm{R}$ script) (Additional file 1: Table S4). Three different genetic models (dominant, recessive and additive effect of single mutated allele) were considered and tested separately. To test confounding effects, individual age and sex was included in regression models as covariates.

\section{Gene expression quantification of potentially causative genes} Expression profiles of four genes (HAO1, BMP2, LOC101117953 and PPP1CC) in 17 tissue types of TBS and MGS were first examined by RT-PCR (Additional file 2: Figure S5). Primer sequences were shown in Additional file 1: Table S5. Samples from TBS and MGS originated from the Dangxiong farm of Tibetan province and Small Tail Han sheep breeding farm of Shandong province, respectively. The sheep were sacrificed by bleeding of the carotid artery for two minutes. 17 tissues were immediately snap-frozen in liquid nitrogen for total RNA extraction. RNA extraction was performed using TRIzol reagent (TaKaRa, Dalian, China). TURBO DNA-free Kit (Ambion, Austin, TX, USA) was used for DNase treatment of total RNA. cDNA for gene amplification and expression was synthesized using PrimeScriptTMRT reagent kit (TaKaRa, Dalian, China). An equal volume of cDNA sample from the same tissue of each of six individuals was pooled for RT-PCR. The reactions were followed according the method described previously [12].

Next, real-time PCR was used to quantify expression levels of BMP2, HAO1 and internal control $\beta$-actin in tissue samples described above. Additional samples of adipose and uterine tissues $(n=8$ per tissue type and tail type) were used for validation of the differential expression of BMP2 gene between tail types. For each measurement, real-time PCR was performed three times and the average gene expression was calculated. Amplification was followed as described in a previous study [12]. Real-time PCR results were processed by the $2^{-\Delta \Delta C t}$ method [51]. The expression of $B M P 2$ in the perirenal adipose tissues of TBS was defined as 1.0 to calculate relative expression levels of four genes.

\section{Neutral test for retro-gene}

Coding sequences of PPP1CC genes (sheep, goat, cattle, human, mouse and clawed frog), as well as retro-PPP1CC genes (sheep and goat) were aligned using MUSCLE algorithm [52] to generate phylogenetic tree. The topology (as showed in Fig. 3b) and codon alignment was treated as the input into PAML [53] "codeml" program to perform a $\log$-ratio test (LRT). In this test, we defined part of the tree as a foreground branch (two r-PPP1CC sequences), and the rest as the background branch (all PPP1CC sequences). $\mathrm{dN} / \mathrm{dS}$ ratio $\omega$ was calculated in these branches separately. To test whether $\omega_{\text {foreground }}$ equaled 1 (which means the evolution of $\mathrm{r}-P P P 1 C C$ putative codons is not different from non-functional sites), we calculated the $\log$-scaled likelihood $(\ln L)$ under model M0 and M1. Under the "null model" $\mathrm{M} 0, \omega_{\text {foreground }}$ was pre-defined as $\omega_{\text {foreground }}=1$; while in M1, $\omega_{\text {foreground }}$ was calculated based on observed substitution rates. Two-fold difference of $\ln L$ between these two models $(2 d \ln L)$ and $P$-value of LRT were then calculated as a statistical measurement (Additional file 1: Table S6).

\section{Additional files}

Additional file 1: Table S1. List of 30-kb windows on MGS genome with top $0.5 \%$ high-LSBL values. Table S2. Information of four genotyped SNPs in association study. Table S3. Genotypes and phenotypes of 116 crossbreeding sheep. Table S4. Linear regression models for genotype-tophenotype association test. Table S5. Primer sequences in the present study. Table 6. Neutral test for retro-PPP1CC gene. (XLS 162 kb)

Additional file 2: Figure S1. Evidence of selective sweep at chromosome 15 near PDGFD. Plots of selective sweep statistics at chromosome 15, from top to bottom: (1) $L S B L$; (2) pair-wise genetic distance $d_{x y}$; (3) intra-lineage heterozygosity $H_{p}$ (standardized). Figure S2. Validation of LOC101117953 DNA sequence. (A) Electrophoresis of PCR product captured by LOC101117953-specific primers. (B) Comparison between the result of PCR product sequencing and the reference assembly of sheep (oviAri3). 
Figure S3. Dot-plots for alignment between eight gene sequences and the sheep genome at chromosome 13. Alignments generated by LASTZ were visualized as dot plots using R script. Exon positions on the normal paralog are showed in blue boxes. Figure S4. Features of different tail types in the hybrid population. Pictures taken for two individual sheep from the hybrid population with thin tail (left) and fat tail (right). Figure S5. Tissue-expression patterns of genes near IBH region. Primers were designed to capture CDNA of five genes from ovine transcriptome. $M$, marker; 1 , pituitary; 2 , hypothalamus; 3 , cerebellum; 4, cerebrum; 5 , ovary; 6, oviduct; 7 . cornua uterus; 8 , corpus uterus; 9 , thyroid; 10 , adrenal gland; 11 , heart; 12 , liver; 13 , spleen; 14 , lung; 15 , kidney; 16 , perirenal adipose 17, tail adipose. Figure S6. Stop-gain mutation on the putative protein sequence of LOC101117953. The position and type of the mutation which truncates the putative protein encoded by LOC101117953 (or r-PPP1CC). The truncated codon is highlighted in red. Figure S7. Chromatin interactions detected in human ESC. Sheep IBH region is homologous to the displayed area on human chromosome 20, between BMP2 and HAO1. Interaction density is visualized by the 3D Genome Browser (http://promoter.bx.psu. edu/hi-c/), using H1-ESC data and a resolution of 10 K. (DOC $1839 \mathrm{~kb})$

\section{Abbreviations}

BMP2: Bone morphogenetic protein 2; EUS: European sheep breeds; HOA1: Hydroxyacid oxidase (glycolate oxidase) 1; IBH region: Intergenic region between BMP2 and HAO1; IROA: Iran sheep; LINE: Long interspersed nuclear element; LSBL: Lineage-specific branch length; LTR: Long terminal repeat; MGS: Chinese Mongolian sheep breeds; MOOA: Northern Africa sheep; PDGFD: Platelet-derived growth factor D; PPP1CC: Protein phosphatase 1 catalytic subunit gamma; GPCR: Quantitative PCR; rPPP1CC: Retro-copy of PPP1CC; RTE: Retro-transposable elements; TBS: Chinese Tibetan sheep breeds

\section{Acknowledgements}

We acknowledge that François Pompanon and Yu Jiang provided the variation data of 20 sheep from Iran (IROA) and 160 sheep from Morocco (MOOA). We thank Emily Aston for editing the English in the manuscript.

\section{Funding}

This work was supported by the Agricultural Science and Technology Innovation Program of China (ASTIP-IAS13), National Natural Science Foundation of China (31802031, 31772580), the Earmarked Fund for China Agriculture Research System (CARS-38), the National Key Technology Support Program (2013BAl101B09), the Doctor Foundation of Linyi University (LYDX2016BS068), Natural Science Foundation of Shandong Province (ZR2018BC045), the National Key Scientific Instrument and Equipment Development Project (2012YQ03026108), the National Basic Research Program of China (2011CB910204, 2011CB510102), the Youth Innovation Promotion Association CAS (2017325). The funders had no role in study design, data collection and analysis, decision to publish, or preparation of the manuscript.

\section{Availability of data and materials}

The 89 genome sequence data used in this study is available on the NCBI sequence archive (SRA) under accession number SRP066883. Variant data for Iranian and Moroccan sheep populations are downloaded from the NextGen project webpage (http://projects.ensembl.org/nextgen/). Targeted genotype and tail phenotype data for 116 cross-breed sheep are provided in the supplementary information for this article.

\section{Authors' contributions}

YXL, MXC, ZYP and SDL conceived this study. ZYP and QYL collected the study materials. SDL and BPM performed population genetic analysis. SDL performed retro-transposon analysis. ZW provided expertise in evolutionary analysis methods. ZYP, XJA and XFG collected genotype and phenotype data for association study. SDL and ZYP performed the regression analysis. ZYP, XJA and XYW performed gene expression experiments and ZYP analyzed the data. YXL and MXC supervised the whole project. ZYP and SDL drafted the manuscript. YXL, MXC, QYL, ZW, ZKZ, RD, WPH, FKL, SJL and DHG revised the manuscript. The final version of manuscript was reviewed and approved by all the authors.

\section{Ethics approval and consent to participate}

All the experimental procedures mentioned in the present study were approved by the Science Research Department (in charge of animal welfare issue) of the Institute of Animal Sciences, Chinese Academy of Agricultural Sciences (IAS-CAAS) (Beijing, China). Ethical approval on animal survival was given by the animal ethics committee of IAS-CAAS (No. IASCAAS-AE-03, 12 December 2016). Consent was obtained from the owners of the animals used in this study.

\section{Consent for publication}

Not applicable.

\section{Competing interests}

The authors declare that they have no competing interests.

\section{Publisher's Note}

Springer Nature remains neutral with regard to jurisdictional claims in published maps and institutional affiliations.

\section{Author details}

${ }^{1}$ Institute of Animal Science, Chinese Academy of Agricultural Sciences, Beijing, China. ${ }^{2}$ Key Lab of Computational Biology, CAS-MPG Partner Institute for Computational Biology, Shanghai Institutes for Biological Sciences, Chinese Academy of Sciences, Shanghai, China. ${ }^{3}$ College of Agriculture and Forestry Science, Linyi University, Linyi, China. ${ }^{4}$ Shanghai Center for Bioinformation Technology, Shanghai Industrial Technology Institute, Shanghai, China.

Received: 11 September 2018 Accepted: 19 March 2019

Published online: 02 April 2019

\section{References}

1. Davidson A. Oxford companion to food. Oxford: Oxford University Press; 1999. p. 290-3.

2. Zhong T, Han JL, Guo J, Zhao QJ, Fu BL, He XH, Jeon JT, Guan WJ, Ma YH. Genetic diversity of Chinese indigenous sheep breeds inferred from microsatellite markers. Small Ruminant Res. 2010;90(1-3):88-94.

3. Tu YR. The sheep and goat breeds in China. Shanghai science and technology press; 1989. p. 6-19.

4. Moioli B, Pilla F, Ciani E. Signatures of selection identify loci associated with fat tail in sheep. J Anim Sci. 2015;93(10):4660-9.

5. Moradi MH, Nejati-Javaremi A, Moradi-Shahrbabak M, Dodds KG, McEwan JC. Genomic scan of selective sweeps in thin and fat tail sheep breeds for identifying of candidate regions associated with fat deposition. BMC Genet. 2012;13:10.

6. Peter C, Bruford M, Perez T, Dalamitra S, Hewitt G, Erhardt G, genetics ECJA. Genetic diversity and subdivision of 57 European and middle-eastern sheep breeds. Anim Genet. 2007;38(1):37-44.

7. Degen AA. Responses to dehydration in native fat-tailed Awassi and imported German mutton merino sheep. Physiol Zool. 1977;50(4):284-93.

8. Atti N, Bocquier F, Khaldi GJAR. Performance of the fat-tailed Barbarine sheep in its environment: adaptive capacity to alternation of underfeeding and re-feeding periods. A review. Anim Res. 2004;53(3):165-76.

9. Kilminster TF, JCJTah G. Production. A note on the reproductive performance of Damara, Dorper and merino sheep under optimum management and nutrition for merino ewes in the eastern wheatbelt of Western Australia. Trop Anim Health Prod. 2011;43(7):1459-64.

10. Frisch RE. Body fat, menarche, fitness and fertility. Hum Reprod. 1987;2(6): 521-33.

11. Wei C, Wang H, Liu G, Wu M, Cao J, Liu Z, Liu R, Zhao F, Zhang L, Lu J. Genome-wide analysis reveals population structure and selection in Chinese indigenous sheep breeds. BMC Genomics. 2015;16(1):194.

12. Pan ZY, Li SD, Liu QY, Wang Z, Zhou ZK, Di R, Miao BP, Hu WP, Wang XY, $\mathrm{Hu} X X$, et al. Whole-genome sequences of 89 Chinese sheep suggest role of RXFP2 in the development of unique horn phenotype as response to semi-feralization. GigaScience. 2018;7(4):giy19.

13. Lv FH, Peng WF, Yang J, Zhao YX, Li WR, Liu MJ, Ma YH, Zhao QJ, Yang GL, Wang $F$, et al. Mitogenomic meta-analysis identifies two phases of migration in the history of eastern eurasian sheep. Mol Biol Evol. 2015; 32(10):2515-33. 
14. Ai H, Fang X, Yang B, Huang Z, Chen H, Mao L, Zhang F, Zhang L, Cui L, He W, et al. Adaptation and possible ancient interspecies introgression in pigs identified by whole-genome sequencing. Nat Genet. 2015;47(3):217-25.

15. Shriver MD, Kennedy GC, Parra EJ, Lawson HA, Sonpar V, Huang J, Akey JM, Jones KW. The genomic distribution of population substructure in four populations using 8,525 autosomal SNPs. Hum Genomics. 2004;1(4):274-86.

16. Sanger F, Coulson AR. A rapid method for determining sequences in DNA by primed synthesis with DNA polymerase. J Mol Biol. 1975;94(3):441-8.

17. Sanger F, Nicklen S, Coulson AR. DNA sequencing with chain-terminating inhibitors. Proc Natl Acad Sci U S A. 1977;74(12):5463-7.

18. Altschul SF, Gish W, Miller W, Myers EW, Lipman DJ. Basic local alignment search tool. J Mol Biol. 1990;215(3):403-10.

19. Hedges SB, Dudley J, Kumar S. TimeTree: a public knowledge-base of divergence times among organisms. Bioinformatics. 2006;22(23):2971-2.

20. Mighell AJ, Smith NR, Robinson PA, Markham AF. Vertebrate pseudogenes. FEBS Lett. 2000;468(2-3):109-14.

21. Xiong Y, Eickbush TH. Origin and evolution of retroelements based upon their reverse transcriptase sequences. EMBO J. 1990;9(10):3353-62.

22. Kordis D, Gubensek F. Unusual horizontal transfer of a long interspersed nuclear element between distant vertebrate classes. Proc Natl Acad Sci U S A. 1998:95(18):10704-9.

23. Deininger PL, Batzer MA. Mammalian retroelements. Genome Res. 2002; 12(10):1455-65.

24. Kordis D, Gubensek F. Horizontal transfer of non-LTR retrotransposons in vertebrates. Genetica. 1999;107(1-3):121-8.

25. Huang HY, Song TJ, Li X, Hu LL, He Q, Liu M, Lane MD, Tang QQ. BMP signaling pathway is required for commitment of $\mathrm{C} 3 \mathrm{H} 10 \mathrm{~T} 1 / 2$ pluripotent stem cells to the adipocyte lineage. Proc Natl Acad Sci U S A. 2009;106(31):12670-5.

26. Kang Q, Song WX, Luo Q, Tang N, Luo JY, Luo XJ, Chen J, Bi Y, He BC, Park $\mathrm{JK}$, et al. A comprehensive analysis of the dual roles of BMPs in regulating adipogenic and osteogenic differentiation of mesenchymal progenitor cells. Stem Cells Dev. 2009;18(4):545 U533.Kang.

27. Ahrens M, Ankenbauer T, Schroder D, Hollnagel A, Mayer H, Gross G. Expression of human bone morphogenetic proteins-2 or -4 in murine mesenchymal progenitor C $3 \mathrm{H} 10 \mathrm{~T} 1 / 2$ cells induces differentiation into distinct mesenchymal cell lineages. DNA Cell Biol. 1993;12(10):871-80.

28. zur Nieden NI, Kempka G, Rancourt DE, Ahr HJ. Induction of chondro-, osteo- and adipogenesis in embryonic stem cells by bone morphogenetic protein-2: effect of cofactors on differentiating lineages. BMC Dev Biol. 2005; 5:1

29. Li QX, Kannan A, Wang W, DeMayo FJ, Taylor RN, Bagchi MK, Bagchi IC. Bone morphogenetic protein 2 functions via a conserved signaling pathway involving Wnt4 to regulate uterine decidualization in the mouse and the human. J Biol Chem. 2007;282(43):31725-32.

30. Lee KY, Jeong JW, Wang JR, Ma LJ, Martin JF, Tsai SY, Lydon JP, DeMayo FJ. Bmp2 is critical for the murine uterine decidual response. Mol Cell Biol. 2007;27(15):5468-78

31. Ying $Y$, Zhao GQ. Detection of multiple bone morphogenetic protein messenger ribonucleic acids and their signal transducer, Smad1, during mouse decidualization. Biol Reprod. 2000;63(6):1781-6.

32. Deckelbaum RJ, Williams CL. Childhood obesity: the health issue. Obes Res. 2012;9(S11):239S-43S.

33. Daniels SR, Morrison JA, Sprecher DL, Khoury P, Kimball TR. Association of body fat distribution and cardiovascular risk factors in children and adolescents. Circulation. 1999:99(4):541.

34. Fox K, Peters D, Armstrong N, Sharpe P, Bell M. Abdominal fat deposition in 11-year-old children. Int J Obes Relat Metab Disord. 1993;17(1):11.

35. Després JP. Body fat distribution and risk of cardiovascular disease an update. Circulation. 2012;126(10):1301-13.

36. Donnelly KL, Smith Cl, Schwarzenberg SJ, Jessurun J, Boldt MD, Parks EJ. Sources of fatty acids stored in liver and secreted via lipoproteins in patients with nonalcoholic fatty liver disease. J Clin Invest. 2005;115(5):1343-51.

37. Cutter AD, Payseur BA. Genomic signatures of selection at linked sites: unifying the disparity among species. Nat Rev Genet. 2013;14(4):262-74.

38. Brosius J. Retroposons - seeds of evolution. Science. 1991;251(4995):753.

39. Kubiak MR, Makalowska I. Protein-coding Genes' Retrocopies and their functions. Viruses. 2017;9(4):80.

40. Han J. Comparative proteomics exploring the biological mechanism of large adipose accumulated in tail or rump of fat-tailed sheep. Beijing: Chinese Academy of Agricultural Sciences; 2016.
41. Hua G-H, Yang L-G. A review of research progress of FecB gene in Chinese breeds of sheep. Anim Reprod Sci. 2009;116(1-2):1-9.

42. Sinclair DC, Mastroyannis A, Taylor HS. Metabolism. Leiomyoma simultaneously impair endometrial BMP-2-mediated decidualization and anticoagulant expression through secretion of TGF- $\beta 3$. J Clin Endocrinol Metab. 2011;96(2):412-21.

43. Souza C, Campbell B, McNeilly A, Baird DJR. Effect of bone morphogenetic protein 2 (BMP2) on oestradiol and inhibin a production by sheep granulosa cells, and localization of BMP receptors in the ovary by immunohistochemistry. Reproduction. 2002;123(3):363-9.

44. Pan Z, Li S, Liu Q, Wang Z, Zhou Z, Di R, Miao B, Hu W, Wang X, Hu X et al. Supporting data for 'Whole-genome sequences of 89 Chinese sheep suggest role of RXFP2 in the development of unique horn phenotype as response to semi-feralization' GigaScience Database 2018.

45. Lamichhaney S, Berglund J, Almen MS, Magbool K, Grabherr M, Martinez-Barrio A, Promerova M, Rubin CJ, Wang C, Zamani N, et al. Evolution of Darwin's finches and their beaks revealed by genome sequencing. Nature. 2015; 518(7539):371-5

46. McKenna A, Hanna M, Banks E, Sivachenko A, Cibulskis K, Kernytsky A, Garimella K, Altshuler D, Gabriel S, Daly M, et al. The genome analysis toolkit: a MapReduce framework for analyzing next-generation DNA sequencing data. Genome Res. 2010;20(9):1297-303.

47. Browning BL, Browning SR. Genotype imputation with millions of reference samples. Am J Hum Gene. 2016:98(1):116-26.

48. Purcell S, Neale B, Todd-Brown K, Thomas L, Ferreira MA, Bender D, Maller J, Sklar P, de Bakker PI, Daly MJ, et al. PLINK: a tool set for whole-genome association and population-based linkage analyses. Am J Hum Gene. 2007; 81(3):559-75.

49. Felsenstein J. PHYLIP - phylogeny inference package (version 3.2). Cladistics. 1989:5:164-6.

50. Harris RS. Improved pairwise alignment of genomic DNA. Ph.D. Thesis: Pennsylvania. The Pennsylvania State University; 2007.

51. Livak KJ, Schmittgen TD. Analysis of relative gene expression data using real-time quantitative PCR and the 2(-Delta Delta $C(T))$ method. Methods. 2001:25(4):402-8.

52. Edgar RC. MUSCLE: multiple sequence alignment with high accuracy and high throughput. Nucleic Acids Res. 2004;32(5):1792-7.

53. Yang Z. PAML: a program package for phylogenetic analysis by maximum ikelihood. Comput Appl Biosci. 1997:13(5):555-6.

\section{Ready to submit your research? Choose BMC and benefit from:}

- fast, convenient online submission

- thorough peer review by experienced researchers in your field

- rapid publication on acceptance

- support for research data, including large and complex data types

- gold Open Access which fosters wider collaboration and increased citations

- maximum visibility for your research: over $100 \mathrm{M}$ website views per year

At BMC, research is always in progress.

Learn more biomedcentral.com/submission 